\title{
Formation, Stability, and Breakup of Nanojets
}

\author{
Michael Moseler and Uzi Landman*
}

\begin{abstract}
Atomistic molecular dynamics simulations reveal the formation of nanojets with velocities up to 400 meters per second, created by pressurized injection of fluid propane through nanoscale convergent gold nozzles with heating or coating of the nozzle exterior surface to prevent formation of thick blocking films. The atomistic description is related to continuum hydrodynamic modeling through the derivation of a stochastic lubrication equation that includes thermally triggered fluctuations whose influence on the dynamical evolution increases as the jet dimensions become smaller. Emergence of double-cone neck shapes is predicted when the jet approaches nanoscale molecular dimensions, deviating from the long-thread universal similarity solution obtained in the absence of such fluctuations.
\end{abstract}

Liquid jets of macroscopic dimensions and their breakup into drops have been of great scientific interest $(1,2)$ and have been used in fuel injection (3), cutting and machining (4, 5 ), spraying and coating, fiber spinning (6), needle and syringe injection, ink-jet printing (7), and micro- and optoelectronic device manufacturing (8). Perturbations at the nozzle opening and surface tension help drive capillary instabilities in jets. Through the application of linear stability analysis to inviscid incompressible fluid columns, Rayleigh concluded that out of all disturbances the fastest growing one has a wavelength of about nine times the column's radius (9). The accepted mechanism of jet breakup involves flow from regions of the liquid column with smaller radii (necks where the Laplace pressure is larger) to crest regions where the pressure is lower, until pinch-off occurs; extensions of Rayleigh's original work have shown $(10-14)$ that the effect of viscosity can reduce the breakup rate and increase the drop size.

The highly nonlinear nature of the NavierStokes (NS) equations that govern fluid flow prevents reliable analyses of the nonlinear dynamics near breakup with linear stability methods or higher order perturbative treatments and requires the development of a full nonlinear theory. Progress in this direction has been achieved with NS simulations (1) and through analytical formulations based on the "lubrication theory" $(1,15-18)$. Particularly pertinent to our study are treatments based on the "slenderness approximation" allowing systematic derivation (1) (starting from the NS equation) of viscous one-dimensional (1D) equations that can be solved numerically with relative ease [unlike the orig-

School of Physics, Georgia Institute of Technology, Atlanta, GA 30332-0430, USA.

*To whom correspondence should be addressed. Email: uzi.landman@physics.gatech.edu inal three-dimensional (3D) NS equation]. Indeed, such treatments have been used recently with remarkable success in investigations of various liquid structures (1), yielding quantitative correct predictions in the neighborhood of breakup, as well as far from it.

Substantial efforts have been invested in studies addressing the design of nozzles (3, $14,18,19)$ including geometrical factors (such as nozzle shape) and material properties (such as wettability of the nozzle by the fluid) and their effects on the efficiency of conversion from potential to kinetic energy (sometimes termed "pressure" and "flow" energies) as well as on the internal and exit flow (velocity) profiles and the magnitude of the disturbances (particularly at the nozzle opening). As the scale of the jets is reduced for use in or fabrication of miniaturized devices, the jet diameter approaches the molecular size, and the commonly used continuum fluid dynamics treatments are of questionable validity $(1,20)$.

We present the results of large-scale atomistic molecular dynamics (MD) simulations (21-23) aimed at extending our knowledge of jet processes to the nanometer-scale regime (that is, to nanojets, or NJs). Although such NJs are envisaged to substantially impact various technologies [such as nanoscale materials' machining and patterning, printing of high-density miniaturized circuitry, and transfer of biological materials (24), such as genes, into cells with minimal damage], understanding the conditions for the formation of NJs and their properties is a most challenging fundamental problem. In particular, because of the reduced dimensions, the behavior of such systems involves large spatiotemporal variations and fluctuations of the liquid properties (such as temperature gradients and liquid density changes caused by viscous heating and evaporative cooling) that complicate (and in many cases invalidate) the use of continuum mechanics treatments and require the development of physically reliable and accurate atomic-scale simulations.

We used a newly developed MD method for atomistic simulations of jet processes to show that NJ formation can indeed be achieved through high-pressure injection and thus overcome large internal viscous energy losses and surface tension effects. We also propose new design principles, such as the use of nonwetting nozzles as well as heating of the nozzle exterior surface, as means for speeding up flow and for enabling NJ formation. Furthermore, aiming at the long-standing goal of relating and integrating atomistic simulations with continuum hydrodynamic modeling, we derive a stochastic modification of the hydrodynamic (deterministic) lubrication equation (LE) for the description of free-surface flow problems and show through comparative MD and hydrodynamic simulations that fluctuation effects increase as the jet radius decreases and that the jet dynamics near breakup is dominated by thermal fluctuations. Our simulations reveal that such fluctuations influence the jet profile near breakup as well as control the breakup length, which we found for NJs to depend only weakly on the strength of perturbations generated in the vicinity of the nozzle just after exit, in contrast to macroscopic jets where the latter processes are the determining factor.

In our MD simulations, we used a common fuel (propane, $\mathrm{C}_{3} \mathrm{H}_{8}$ ) as a fluid confined and injected into vacuum through a nozzle (25-30). Various configurations of the inlet nozzle (modeled as crystalline metal) were used, including a long tube of constant diameter, a hole in the wall of the container, and a convergent nozzle (31); below, we show results for the convergent nozzle (Fig. 1A).

To determine the conditions for jet formation, we performed a number of simulations for a range of exit orifice diameters $D$ between 2 and $6 \mathrm{~nm}$ and nozzle inlet pressures $P_{0}$ between 250 and $500 \mathrm{MPa}$ (the upper limit was chosen to prevent glassification of the propane fluid), with an overall nozzle temperature $T_{0}=150 \mathrm{~K}$. A steady-state jet (with a velocity of $200 \mathrm{~m} / \mathrm{s}$ ) was produced (32) only in simulations with $P_{0}=500 \mathrm{MPa}$ and with the gold atoms of the outer surface of a 6-nm-diameter nozzle heated to the boiling temperature of propane $(230 \mathrm{~K})$ while the temperature of the inside gold atoms was kept at $T_{0}=150 \mathrm{~K}$. Under these conditions, the thickness of the wetting film on the outer surface of the nozzle (Fig. 1A, time $t \geq 20$ ps) was limited to three propane layers. Without heating of the outer surface, a transient initial jet formed, but it was slowed down because of viscous frictional interactions with a thickening wetting film, as well as by surface tension effects, culminating in blocking of the flow. Jet formation was also inhibited for $P_{0}=500 \mathrm{MPa}$ when smaller orifice 
diameters were used $(D<6 \mathrm{~nm})$ because of a strong pressure drop caused by frictional losses inside the nozzle, and also for the 6-nm-diameter orifice with a smaller driving pressure $P_{0}=250 \mathrm{MPa}$. As an alternative to preventing formation of thick wetting blocking films by heating of the outside surfaces of the nozzle as described above, we found for the 6-nm-diameter nozzle with $P_{0}=500$ $\mathrm{MPa}$ and $T_{0}=150 \mathrm{~K}$ that a jet can be formed (with a higher velocity of $440 \mathrm{~m} / \mathrm{s}$ ) under nonwetting conditions, which we modeled by truncating the interaction potential between the propane molecules and the exterior gold atoms of the nozzle to exclude the attractive interaction between them (in practice, one could use a nonwetting thin coating applied to the exterior of the nozzle).

Atomistic configurations during the initial formation stages of the jet (with heated outside surfaces of the nozzle exit) are shown in Fig. 1A, and the evolution of the jet during the first 2 ns of the simulation is shown in Fig. 1B. The exit of the fluid from the nozzle is accompanied by evaporation of molecules and lateral (radial) spreading resulting in thin-film formation, accompanied by thickening (swelling) of the fluid column (Figs. 1A and $2 \mathrm{~A}$ ); a much reduced swelling of the jet was found in the nonwetting simulation (Fig. 2B). In both cases, the velocity profile (33) is perturbed at the exit (larger perturbation in the wetting case) and relaxes over a rather short distance $(5 \mathrm{~nm}$ for the wetting case and a shorter distance for the nonwetting one), transforming from laminar flow (34), with a parabolic velocity profile inside the nozzle, to a uniform velocity profile (pluglike) outside (Fig. 2, A and B).

The variations with distance along the jet of the time-averaged axial $\left(v_{z}\right.$, along the jet axis) and radial ( $v_{\mathrm{r}}$, perpendicular to the axis) velocities of the jet calculated at steady state for $z \leq L_{\mathrm{B}}$ (where $L_{\mathrm{B}}$ is the average intact length of the jet, also called breakup length) are displayed in Fig. 2C. In both the wetting (solid lines) and nonwetting (dashed lines) cases, $v_{z}$ increases in the converging section $(z \leq-1 \mathrm{~nm}$ ) of the nozzle (as expected because of the continuity of the fluid), and the increase of $v_{\mathrm{r}}$ reflects focusing of the flow; however, for the wetting case, near the nozzle exit the attractive interactions of the propane molecules with the nozzle surfaces cause a substantially smaller increase in $v_{z}$ and a somewhat larger increase in $v_{\mathrm{r}}$, resulting in drag forces on the flowing fluid. Upon exit, the internal pressure and density in the liquid drop precipitously (Fig. 2D). The pressure in the fluid after the nozzle remains constant and near zero, whereas the density shows a slow gradual increase that is related to evaporative cooling of the jet.

Internal frictional processes increase the kinetic temperature of the molecular fluid substantially in the nozzle exit region. In the wetting case, friction occurs even outside the nozzle because of the molecular film adsorbed on the exterior surface of the nozzle, and consequently, the temperature is further increased (Fig. 2E). Immediately past the nozzle (for $z \leq 20 \mathrm{~nm}$ ), the total internal energy of molecules near the surface of the jet is positive (gray areas in Fig. 2E), leading to a decrease in the temperature due to efficient evaporative cooling and consequent reduction in the jet radius along the jet axis; the rate of these processes slows for $z \geq 30 \mathrm{~nm}$.

The initial (transient) stage of the evolution of the jet (Fig. 1B, $t<1 \mathrm{~ns}$ ) is characterized by the generation of small fast-moving droplets and molecular clusters, portrayed by sawtoothshaped discontinuities in $L$ at the droplets' detachment times (Fig. 1B, inset), where $L$ is the intact length of the jet. At steady state ( $t \geq 1$ $n s)$, the jet instabilities result in the formation of droplets with a narrow size distribution corresponding to an average intact length of the jet (breakup length) $L_{\mathrm{B}} \approx 170 \mathrm{~nm}$ (we also obtained similar $L_{\mathrm{B}}$ values in simulations with a hotter propane fluid, $T_{0}=200$ and $250 \mathrm{~K}$ ); also, a smaller satellite droplet that eventually merged with the preceding larger one occasionally formed (Fig. 1B, $t=1 \mathrm{~ns}$; Fig. 3C, $t=1.65$ ns). Typically, the neck shape before pinch-off resembles a double cone (see Fig. 3B and the
Fig. 1. (A) Atomic configurations selected from MD simulations of a propane $\mathrm{NJ}$ formed through injection into vacuum through a convergent 3D gold nozzle with the dimensions given in the top left panel. The upper solid gold walls were removed to expose the interior of the nozzle. A pressure of $500 \mathrm{MPa}$ is applied at the back of the nozzle assembly, the temperature inside the nozzle is controlled at $150 \mathrm{~K}$, and the exterior gold surface of the nozzle exit is heated to $230 \mathrm{~K}$. The sequence of configurations depicts the initial exit of the propane fluid (10 ps) and wetting of the outside surface of the nozzle to form a thin adsorbed propane film, accompanied by swelling of the exiting fluid propane jet near the nozzle exit ( $t \geq 20 \mathrm{ps})$. The formation of the jet, which achieves a flow velocity of $200 \mathrm{~m} / \mathrm{s}$, is accompanied by evaporative cooling, and steady-state flow is achieved at $\sim 1 \mathrm{~ns}$. The propane molecules are depicted in blue, and the gold atoms are in yellow. (B) Evolution of the propane $\mathrm{NJ}$ along the direction of propagation ( $\mathrm{z}$ axis) at selected times ( $0.1 \mathrm{~ns} \leq t \leq 2 \mathrm{~ns}$ ) after exit from the nozzle. Formation of fast-moving droplets and molecular clusters is observed at the initial (transient) stage ( $t \leq 1 \mathrm{~ns})$, achieving steady-state flow conditions $(v=200 \mathrm{~m} / \mathrm{s})$
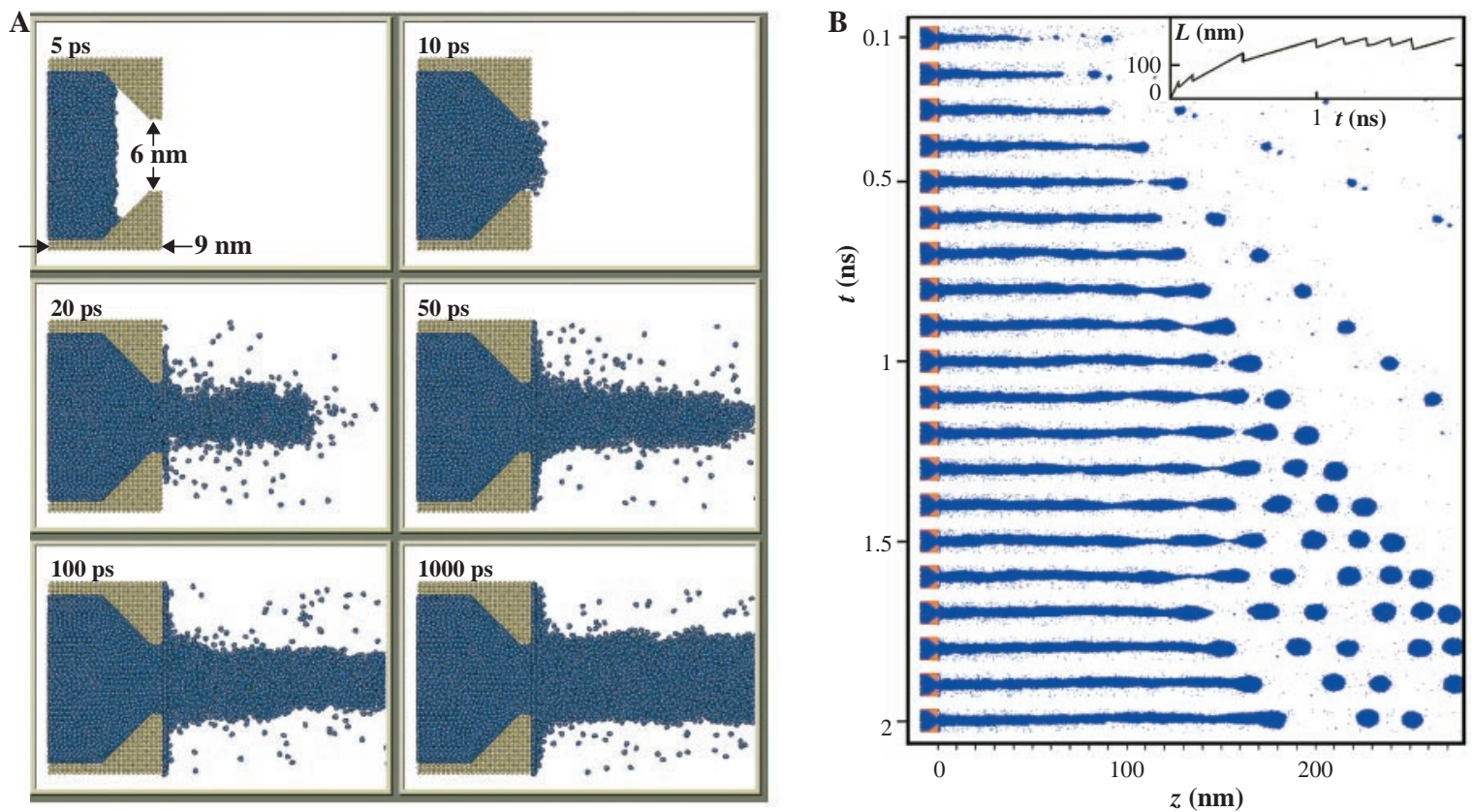

at $t \approx 1$ ns. Molecular evaporation and formation of necking instabilities are observed, resulting in breakup events and formation of drops. At pinch-off, the droplets are of elongated ellipsoidal shape, and they become rounded shortly after. On some occasions, small satellite (secondary) drops are seen (for example, at $t=1 \mathrm{ns)} \mathrm{merging} \mathrm{later} \mathrm{with} \mathrm{the} \mathrm{preceding} \mathrm{larger} \mathrm{drop.} \mathrm{In}$ the inset, we display the time evolution of the intact length of the jet. Each sawtooth-shaped discontinuity corresponds to a pinch-off of a drop. At $t \geq$ $1 \mathrm{~ns}$, steady state is achieved with the mean breakup length of the jet, $L_{\mathrm{B}} \approx$ $170 \mathrm{~nm}$. 
top configuration in Fig. 3D for the wetting and nonwetting cases, respectively); occasionally, the jet profile deviates from axisymmetry (Fig. $3 \mathrm{~A})$, or it may be somewhat elongated, forming a small cluster between the intact part and the main droplet (Fig. 3C). The neck radius decreases steadily to its final value of one or two molecular diameters, and a smooth separation of the few remaining molecules in the doublecone apex completes the pinch-off process.

In the hydrodynamic description $(1,18)$, the radius of the neck shrinks as breakup of the jet is approached, and the fluid velocity in this region increases strongly (developing a singularity at the pinch-off time), thus localizing the breakup process both spatially and temporally $(1,35)$. Such "locality" is reflected in the independence of the fluid motion near breakup from the initial conditions and from details of the experimental setup (e.g., the radius of the nozzle); that is, the fluid motion near pinch-off becomes universal (1). Under these circumstances, the only relevant length and time scales $(1,18,35)$ are $l_{\eta}=\eta^{2} /(\rho \gamma)$ and $t_{\eta}=\eta^{3} /\left(\rho \gamma^{2}\right)$ (expressing the balance between viscous and surface tension forces), respectively, which are determined by the viscosity $\eta$, the density $\rho$, and the fluid surface tension $\gamma$. Furthermore, asymptotic analysis (1) reveals that the fluid motion close to the breakup singularity is a similarity solution of the equations of motion (see below), with the jet profile consisting of long thin threads connected by strongly sloped segments to the rest of the fluid.

In general, the neck shape of macroscopic jets $(1,35)$ depends on the ratio between the observation scale $l_{\text {obs }}$ and the intrinsic material-dependent length scale $l_{\eta}$. For $l_{\text {obs }} / l_{\eta} \gg$ 1 , the shape of the neck near the pinch point approaches that of a cone connected to a spherical cap (35), whereas for $l_{\text {obs }} / l_{\eta}<1$, the aforementioned similarity solution thinthread profile occurs (1); for macroscopic values of $l_{\mathrm{obs}}$ [that is, $l_{\mathrm{obs}} \sim 1 \mu \mathrm{m}$, which is the limit for optical detection (20)], the above classes of behavior are associated with fluids whose viscosities are low (such as water) or high (such as glycerol), respectively. For the propane NJ (with $l_{\text {obs }}$ of the order of the neck radius, that is, a few nanometers), this ratio is $<1$ (even though propane is a low-viscosity fluid), and thus, thin-thread formation is expected and indeed confirmed (Fig. 3D, LE simulation) by numerical integration (36) of the LE simulations ( 1 ) for the axial velocity $v(z, t)$ and the radius $h(z, t)$ of the jet along the $z$ axis

$$
\begin{gathered}
\partial_{t} v(z, t)+v(z, t) \partial_{z} v(z, t)=-\left[\partial_{z} p(z, t)\right] / \rho \\
+3 v \partial_{z}\left[h(z, t)^{2} \partial_{z} v(z, t)\right] / h(z, t)^{2} \\
\partial_{t} h(z, t)+v(z, t) \partial_{z} h(z, t) \\
=-\left[\partial_{z} v(z, t)\right] h(z, t) / 2
\end{gathered}
$$

with the Laplace pressure given by

$$
\begin{aligned}
& p(z, t)= \gamma \kappa(z, t)= \\
& \gamma\left(\frac{1}{h(z, t)\left\{1+\left[\partial_{z} h(z, t)\right]^{2}\right\}^{1 / 2}}-\right. \\
&\left.\frac{\partial_{z}^{2} h(z, t)}{\left\{1+\left[\partial_{z} h(z, t)\right]^{2}\right\}^{3 / 2}}\right)
\end{aligned}
$$

where $\kappa$ is the surface curvature and $\nu=\eta / \rho$ (in Eq. 1) is the kinematic viscosity.

However, the long thread-shaped neck

(Fig. 3D, middle), the mean breakup length of $L_{\mathrm{B}} \approx 530 \mathrm{~nm}$ (Fig. 3E, top), and the steady-state variation with time of the smallest neck radius of the jet (minimal jet radius $h_{\text {min }}$ in Fig. 3E, bottom) obtained through the LE simulations are in obvious disagreement with the corresponding MD simulation results, which exhibit a double-cone (sometimes called "hourglass") neck shape (Fig. $3 \mathrm{D}$, top), a much smaller mean breakup
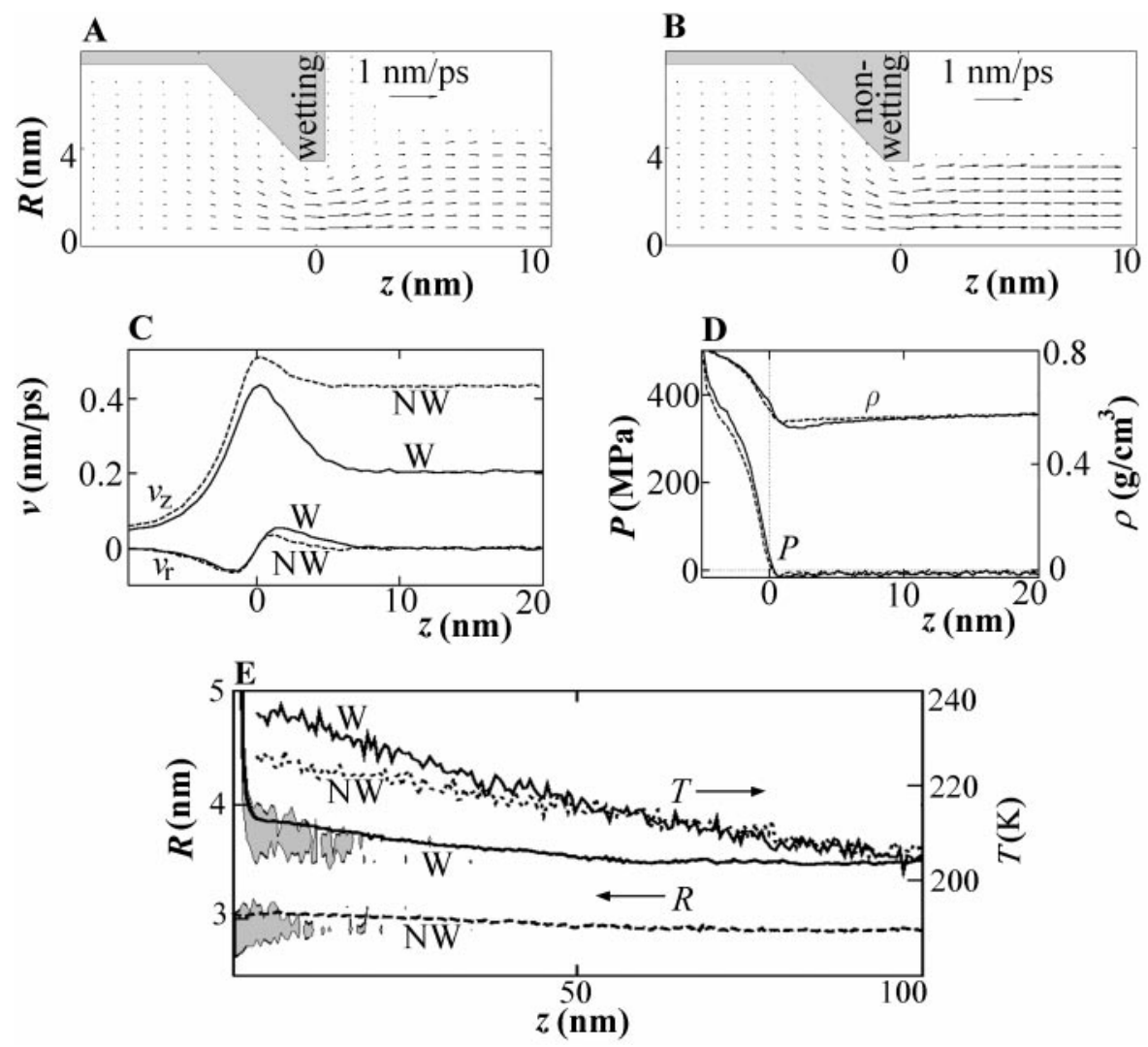

Fig. 2. Steady-state properties of the propane $\mathrm{NJ}$ formed by injection of propane through a nozzle with a 6-nm diameter. Velocity profiles of the propane fluid are shown for simulations (A) where the propane wets the exterior surface of the nozzle and (B) where the exterior surfaces of the nozzle are nonwetting. At the nozzle exit $(z=0)$, the flow-velocity profile is laminar with a parabolic variation (as shown by the length of the arrows) from the solid gold surface to the middle. Formation of an adsorbed film (with a thickness of three propane layers) in the wetting case causes swelling of the exiting jet with a rapid relaxation to a pluglike flow profile [at $z \sim 5 \mathrm{~nm}$ in (A)]. Minimal swelling is found in the nonwetting case (B). The steady-state flow velocities of the $\mathrm{NJ}$ are 200 and $440 \mathrm{~m} / \mathrm{s}$ for the wetting and nonwetting cases, respectively. (C) Time-averaged axial $\left(v_{z}\right)$ and radial $\left(v_{r}\right)$ velocities of the jet (calculated as an average over the corresponding velocities of the propane molecules in cylindrical slices with a radius of $2 \mathrm{~nm}$ and a width of $0.5 \mathrm{~nm}$ centered on the jet axis) versus distance along the $z$ axis for the wetting (W) (solid lines) and nonwetting (NW) (dashed lines) cases. There is a larger increase in $v_{z}$ upon exit and a relaxation to a higher flow velocity for the NW nozzle. (D) Time-averaged variation of the pressure $P$ and density $\rho$ of the propane fluid plotted versus $z$, exhibiting sharp drops in the convergent $(z \leq 0)$ part of the nozzle. (E) Time-averaged variations of the propane $\mathrm{NJ}$ radius $R$ (bottom two curves) and molecular kinetic temperature $T$ (upper curves) for the W (solid lines) and the NW (dashed lines) cases; $T$ is calculated by subtracting the local flow velocity of the jet from the instantaneous molecular velocities and time-averaging the resultant molecular kinetic energies in slices of the jet along the $z$ axis. The emanating jet has a higher temperature for the wetting case with $T$ going above the boiling point of propane $(230 \mathrm{~K})$ right after the exit. With efficient cooling of the emanating "gaseous fluid" through molecular evaporations from the surface of the jet, which is accompanied by gradual shrinking of the jet radius, $T$ decreases and the propane density in the jet increases $[\rho$ in (D)], causing condensation of the propane fluid. The gray areas superimposed on the radius plots indicate spatial regions where the total internal energy (potential plus molecular kinetic energy) of the propane molecules is positive, corresponding to regions of the surface layers of the jet where molecular evaporation is most probable. 
length $L_{\mathrm{B}} \approx 250 \mathrm{~nm}$ (Fig. 3E, top), and a manifestly less regular variation of $h_{\text {min }}$ that exhibits faster decay near pinch-off (Fig. 3E, bottom). These observations indicate the inapplicability of the deterministic LE continuum treatment for the description of nanoscopic jets and of nanoscale free-surface flow problems in general with a likely source of the difficulty being the neglect of fluctuations $(1,20,37,38)$. Indeed, the continuum description of such small systems requires the use of exceedingly small volumes, each containing a very limited number of particles, and consequently, continuum variables associated with such small volume elements, which represent (local) averages over the properties of the microscopic constituents, are expected to exhibit large fluctuations.

To extend the LE continuum approach to the nanoscale regime, we derived a stochastic version of the LE (SLE) by applying the slenderness approximation to the NS equation $\rho\left(\partial_{t} v_{i}+\Sigma_{j} v_{j} \partial_{j} v_{i}\right)=\Sigma_{j} \partial_{j} \sigma_{j i}$ (here, $i$ and $j$ are coordinate indices $i, j=1,2$, and 3 ), with a Gaussian noise obeying the fluctuationdissipation theorem (FDT) $(39,40)$ added to the deterministic stress tensor $\sigma_{i j}$. In the resulting SLE, the dynamics of the jet is described by Eqs. 1 to 3 with a term $-\partial_{z} f /$ $\left(\pi h^{2} \rho\right)$ added to the right-hand side of Eq. 1, where $f$ is a Gaussian white-noise variable obeying the FDT $\left\langle f(z, t) f\left(z^{\prime}, t^{\prime}\right)\right\rangle=$ $6 k_{B} T \eta \pi h^{2}(z, t) \delta\left(z-z^{\prime}\right) \delta\left(t-t^{\prime}\right) ; k_{\mathrm{B}}$ is the Boltzmann constant. The magnitude of the added stochastic term increases as the lateral dimension of the jet (the radius $h$ ) decreases.

The results of simulations of the propane NJ using the SLE are in remarkable agreement with the MD simulations, as evident from comparison of the neck shapes (Fig. 3D), the breakup length (Fig. 3E, top), and the evolution of $h_{\text {min }}$ (Fig. 3E, bottom). In the deterministic LE solution, the minimum neck radius decays (close to pinch-off) linearly in time (measured from the pinch-off time $t_{0}$ ); that is, $h_{\min }=$ $0.03 l_{\eta}\left(t-t_{0}\right) / t_{\eta}$, following the universal law associated with the similarity solution (1), whereas both the SLE and the MD simulations exhibit a less regular pattern of $h_{\min }$ versus $t$ and a faster decay [for results obtained with MD, LE, and SLE comparative simulations pertaining to breakup processes in a liquid bridge at constant temperature, as well as a discussion of the effects due to the disjoining pressure (41), see the supplementary material (42)].

The above results suggest that in NJs the very nature of the dynamical evolution is influenced strongly by hydrodynamic fluctuations, deviating in a substantial way from the behavior predicted through the analysis of the deterministic LE. To further elucidate the role of such fluctuations on the dynamical evolution, we rewrite the force balance equation of the SLE (that is, Eq. 1 with the aforementioned added term) in dimensionless form

$$
\begin{gathered}
\partial_{t} v+v \partial_{z} v=-\left(\partial_{z} \kappa\right)+3 \partial_{z}\left(h^{2} \partial_{z} v\right) / h^{2} \\
-M_{l} \sqrt{6 / \pi} \frac{\partial_{z} h N}{h^{2}}
\end{gathered}
$$

with all quantities expressed in units of $l_{\eta}$ and $t_{\eta}, N$ denoting a standard Gaussian (white) stochastic process, and $M_{l}=l_{T} / l_{\eta}$, where $l_{T}$ $=\sqrt{k_{\mathrm{B}} T / \gamma}$ is the thermal capillary length. For most materials, $l_{T}$ is of the order of interatomic distances and is smaller than $l_{\eta}$; thus, $M_{l}<1$.

As mentioned above, a key concept, which emerged first from experimental (photographic) (35) studies motivated by early theoretical work (43) on the shape of a liquid bridge close to bifurcation (that is, separation of a nascent drop from its parent body), is that of similarity solutions $(1,35)$ and the associated classification of the neck shapes according to the ratio $l_{\text {obs }} / l_{\eta}$; interestingly, $\sqrt{l_{\eta} / l_{\text {obs }}}=O h$, where $O h$ is the Ohnesorge number occurring often in viscous free-surface flow problems $(14,18)$. The appearance of an additional length scale in the SLE simulations (that is, $l_{T}$ entering Eq. 4 through the number $M_{l}$ ) is a direct consequence of the extension to include temperature-dependent stress fluctuations, and its magnitude determines the nature of the jet evolution, including the appearance of solutions other than the universal ones predicted through the deterministic LE (1).

For example, it is known that when the observation scale $l_{\text {obs }}$ (which we take here to be of the order of the neck radius) is in the range $l_{\text {obs }} \gg l_{\eta}$, cap-cone neck shapes occur (35), whereas when $l_{\text {obs }}$ is less than $l_{\eta}$ but still substantially larger than $l_{T}$ (that is, $M_{l} \ll 1$ ), thread formation will be observed for several decades of reduction in $l_{\text {obs}}$; this is the case

Fig. 3. (A through C) Selected atomistic configurations obtained by $M D$ simulations with a wetting 6-nmdiameter nozzle, illustrating various breakup scenarios. In each frame, two consecutive times are shown. The most frequently observed breakup process, exhibiting close to pinch-off formation of an axisymmetric double-cone shape of the neck, is displayed in (B). Also shown is the occasional formation of a nonaxisymmetric neck configuration (A) and generation of a somewhat elongated neck resulting in the split-off of a small cluster (C). (D)

A B
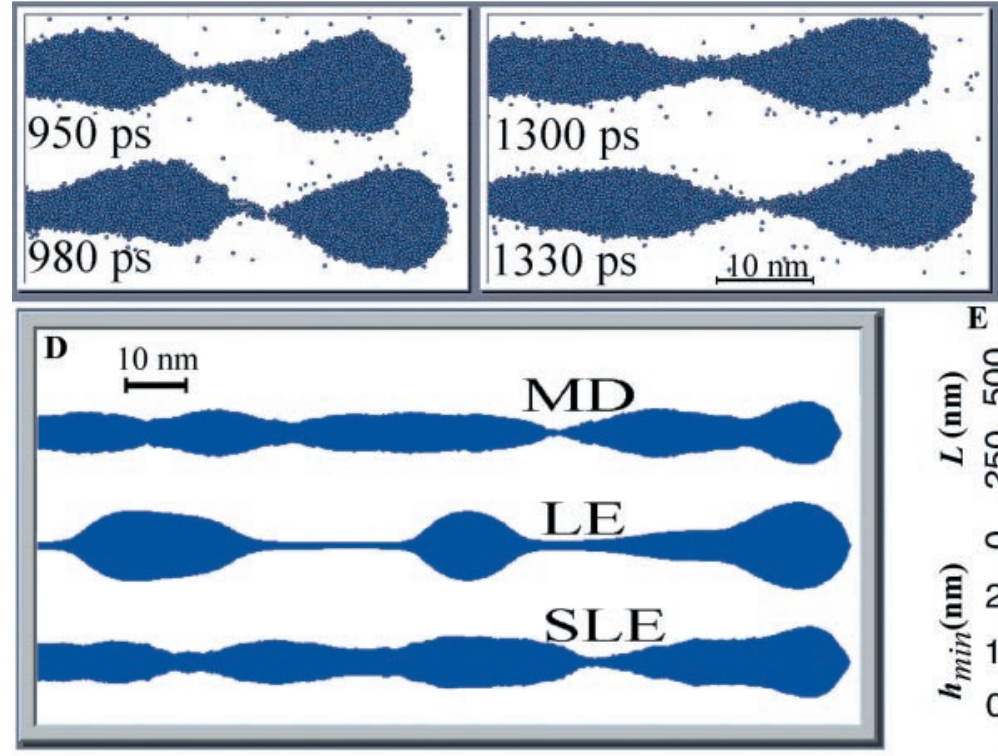

C

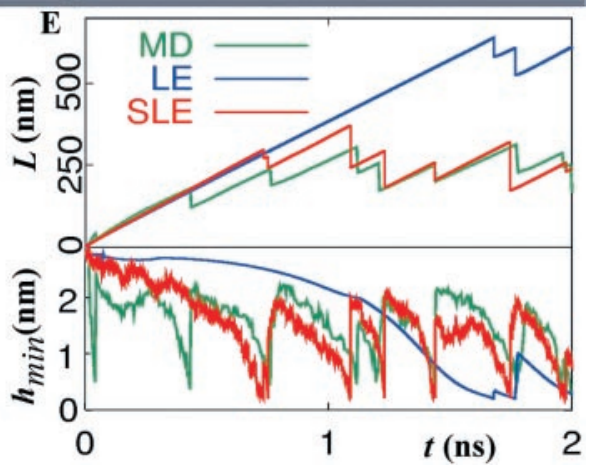

Results pertaining to

breakup events of the propane jet for a 6-nm-diameter nonwetting nozzle were obtained by the following: an atomistic MD simulation, a simulation using the deterministic LE formulation (Eqs. 1 to 3), and a simulation using the SLE (Eqs. 1 to 3, with the modification of Eq. 1 to include fluctuating stresses). Double-cone neck shapes are exhibited in the MD and SLE simulations, in contrast to the long thread obtained with the LE simulation. (E)
Results for the time evolution of the intact jet length $L$ (upper curves) and for the minimal jet radius $h_{\min }$ (bottom curves) obtained from MD, LE, and SLE simulations (distinguished by color). Each of the sawtooth-shaped discontinuities in $L$ and corresponding sharp dips in $h_{\min }$ are associated with a breakup event resulting in the formation of a drop. There is remarkable agreement between the MD and SLE results. 


\section{R E P O R T S}

for glycerol, where at $300 \mathrm{~K}, l_{T}=0.26 \mathrm{~nm}$, $l_{\eta}=3.9 \times 10^{-2} \mathrm{~m}$, and $M_{l}=0.66 \times 10^{-8}$. Of course, as $l_{\text {obs }}$ is decreased, the role of the fluctuations increases and results in the appearance of a hierarchy of secondary necks on the threads (20). However, when $l_{\text {obs }}$ approaches microscopic dimensions (i.e., $l_{\text {obs }} \sim l_{T}$ ) the fluctuations will dominate the breakup process and double-cone neck shapes will appear (Fig. 3D, SLE simulation) (44). For fluids with $M_{l} \sim$ 1 (e.g., the model propane fluid used in our simulations, where at $210 \mathrm{~K}, l_{T}=0.56 \mathrm{~nm}$, $l_{\eta}=2.3 \mathrm{~nm}$, and $\left.M_{l}=0.24\right)$, the range of $l_{\mathrm{obs}}$ values corresponding to thread formation is exceedingly narrow, and consequently, a direct transition with decreasing $l_{\text {obs }}$ between the cone-cap to the double-cone neck shapes is predicted, as is observed in our MD and SLE simulations. In intermediate cases (that is, $M_{l}<$ 1 but not $M_{l} \ll 1$, as in water, where at $300 \mathrm{~K}$, $l_{T}=0.24 \mathrm{~nm}, l_{\eta}=13.7 \mathrm{~nm}$, and $M_{l}=0.018$ ), we expect a tendency for thread formation at $l_{\text {obs }} \approx 10 \mathrm{~nm}$, but unlike the case of fluids with $M_{l} \ll 1$ (e.g., glycerol), this stage is predicted to transform to the double-cone regime early on with a further decrease in $l_{\text {obs }}$ (that is, for a neck radius of $\sim 1 \mathrm{~nm}$ ), without the development of a hierarchy of secondary necks on the threads (as in the glycerol case mentioned above).

\section{References and Notes}

1. J. Eggers, Rev. Mod. Phys. 69, 865 (1997).

2. The review in (1) contains original material as well as a comprehensive discussion and list of references to earlier work on dynamics and breakup processes in liquid jets and other free-surface flow problems.

3. K. Kuo, Ed., Recent Advances in Spray Combustion: Spray Atomization and Drop Burning Phenomena (American Institute of Aeronautics and Astronautics, Reston, VA, 1996), vols. 1 and 2.

4. D. A. Summers, Waterjetting Technology (Spon, London, 1995).

5. K. Yamaguchi, in Proceedings of the 1997 International Symposium on Micromechatronics and Human Science (Institute of Electrical and Electronics Engineers, Piscataway, NJ, 1997), pp. 33-40.

6. A. L. Yarin, Free Liquid Jets and Films: Hydrodynamics and Rheology (Longman, Essex, UK, 1993).

7. See the review by R. W. Kenyon [in Chemistry and Technology of Printing and Imaging Systems, P. Gregory, Ed. (Blackie, London, 1996), pp. 113-138]

8. D. J. Hayes, W. R. Cox, M. E. Grove, J. Electron. Manuf. 8, 209 (1998).

9. J. W. S. Rayleigh, Proc. R. Soc. London 10, 4 (1878). 10. Philos. Mag. 34, 145 (1892)

11. C. Weber, Z. Angew. Math. Mech. 11, 136 (1931).

12. S. Chandrasekhar, Hydrodynamic and Hydrodynamic Stability (Oxford Univ. Press, New York, 1961)

13. See the review by S. P. Lin and R. D. Reitz [Annu. Rev. Fluid Mech. 30, 85 (1998)].

14. M. J. McCarthy and N. A. Maloy, Chem. Eng. J. 7, 1 (1974).

15. H. C. Lee, IBM J. Res. Dev. 18, 364 (1974).

16. S. E. Bechtel, M. G. Forrest, K. J. Lin, Stab. Appl. Anal. Contin. Media 2, 59 (1992).

17. J. Eggers and T. F. Dupont, J. Fluid Mech. 262, 205 (1994).

18. S. Middleman, Modeling Axisymmetric Flows (Academic Press, New York, 1995).

19. Y. G. Chesnokov and N. A. Razumovskij, Appl. Sci. Res. 59, 77 (1998).

20. X. D. Shi, M. P. Brenner, S. R. Nagel, Science 265, 219 (1994).

21. J. Koplik and R. Banavar, Phys. Fluids A 5, 521 (1992).

22. S. Kawano, Phys. Rev. E 58, 4468 (1998).
23. In the early simulations in (21), systems with a relatively small number of Lennard-Jones particles were used to investigate stability issues of liquid threads [see also (22)]. In addition, the burst of a liquid drop immersed in a second liquid undergoing shear and the rupture of a liquid sheet were considered. Formation, stability, and breakup processes of liquid jets were not studied in (21) and (22).

24. For a review on gene transfer by biolistic processes, see work by P. A. Furth [Mol. Biotechnol. 7, 139 (1997)

25. The propane molecules were modeled after (26) and supplemented by angle-bending potentials following (27). For the gold nozzle atoms, we used a manybody embedded-atom interaction (28). The interaction potentials between the propane and the metal atoms were adopted from (29).

26. W. L. Jorgenson, J. D. Madura, C. J. Swenson, J. Am Chem. Soc. 106, 6638 (1984)

27. P. Ploeg and H. J. C. Berensen, J. Chem. Phys. 76, 3271 (1982)

28. D. J. Oh and R. A. Johnson, J. Mater. Res. 3, 471 (1988)

29. T. K. Xia, J. Ouyang, M. W. Ribarsky, U. Landman, Phys. Rev. Lett. 69, 1967 (1992)

30. To simulate a continuous supply of propane to the nozzle assembly, we first prepared a 14-nm-diameter, 128-nm-long cylinder of propane, containing $\sim 200,000$ molecules that were equilibrated initially at $P_{\mathrm{i}}=500 \mathrm{MPa}$ and $T_{\mathrm{i}}=150 \mathrm{~K}$ and then frozen instantaneously into an arbitrary liquid configuration. At the start of the simulation, the inlet part of the nozzle assembly was filled with the top part of the frozen propane cylinder, and the dynamics of the propane molecules in the nozzle inlet as well as of the nozzle metal atoms were turned on with Langevin temperature control to the desired temperature $T$ above the melting point of propane (Fig. 1A, top left) At the same time, a force $A P_{0}$ (where $A$ is the cross-sectional area of the cylinder and $P_{0}$ is the chosen driving pressure) was applied at the back of the propane cylinder, and the force acting in the opposite direction from the liquid propane on the frozen cylinder was calculated. The resultant tota force was then calculated and used to move the whole frozen propane according to Newton's equations of motion with an effective mass of 1000 molecules. As the propane cylinder was pushed into the back of the nozzle, the dynamics of molecules of the propane cylinder entering a 0.4-nm-thick zone at the nozzle inlet was also turned on with thermalization to $T_{0}$. In this way, the propane is forced through the nozzle outlet (Fig. 1A, at $t \geq 10 \mathrm{ps}$ ), and to maintain the prescribed pressure at the inlet, new molecules enter the nozzle in a continuous manner. The simulation zone past the nozzle exit consisted of a rectangular box of dimensions $16 \mathrm{~nm}$ by $16 \mathrm{~nm}$ by $340 \mathrm{~nm}$ (the long dimension in the direction of the propagative jet), and molecules leaving this region were removed from the simulation. With a loadbalanced parallelized code (employing an atom decomposition technique), a typical simulation lasted 2 $\mathrm{ns}$, involving the dynamic evolution of $\sim 100,000$ propane molecules and 17,000 nozzle metal atoms.

31. The nozzle (Fig. 1A) was modeled as an inlet tube with a 14-nm inner diameter and 4-nm length leading to a 4-nm-long convergent section (with an angle of $135^{\circ}$ between the straight part of the inlet tube and the sloping convergent part) and culminating in an orifice of diameter $D$ and a length of $1 \mathrm{~nm}$. The two innermost atomic layers of the metal (gold) surrounding the whole nozzle (including the top layers of the surface of the metal at the outlet) were treated dynamically and were thermally controlled to the desired temperature through Langevin dynamics. The other metal atoms in the metal block were held statically at their crystalline lattice positions.

32. Consideration of the balance between the inertia force and the surface tension force of a fluid emanating from a nozzle, namely $\pi R^{2} \rho v^{2} / 2=2 \pi R \gamma$, where $\rho$ and $\gamma$ are the density and surface tension of the fluid, respectively, and $R$ is the radius of the nozzle (assuming that the width of the emanating liquid is the same as that of the nozzle opening) leads to a criterion for the velocity corresponding to jet formation (i.e., $v>v_{c}$, with $v_{c}$ defined by the above equation as the critical velocity), which can be expressed in terms of a critical Weber number We $=$ $\rho v_{c}^{2} 2 R / \gamma=8$ [see (13)]. The velocity of the emanating fluid may be estimated from the (pipe flow) HagenPoisseulle law $v=R^{2} P_{0} /(8 \eta L)$ [see (18)], where $P_{0}$ is the applied pressure, $\eta$ is the fluid viscosity, and $L$ is the effective length of the nozzle, which we take as $=I R$. Combining the critical Weber number criterion with the Hagen-Poisseulle law results in an expression for the critical pressure for jet formation $P_{c}=16 \eta l(\gamma / \rho)^{1 / 2} / R^{3 / 2}$. Using $l \approx 1, \gamma=0.009 \mathrm{~N} / \mathrm{m}$ see (36)], and $\eta=7.2 \times 10^{-4} \mathrm{~N} \cdot \mathrm{s} / \mathrm{m}^{2}$ (calculated through a separate MD simulation at $T=150 \mathrm{~K}$ and $1 \mathrm{~atm})$ for our propane model fluid, we estimate that the critical pressures for $R=1,2$, and $3 \mathrm{~nm}$ are 1400 500 , and $270 \mathrm{MPa}$, respectively. These simple estimates correlate well with the results of our MD simulations.

33. After a short transient period (typically $<1 \mathrm{~ns}$ ) steady-state conditions were established; in analyzing results of the simulations, the transient period was discarded and only steady-state properties were recorded.

34. In our system, the Reynolds number Re is small; that is, $\operatorname{Re}=13$.

35. D. H. Peregrine, G. Shoker, A. Symon, J. Fluid Mech. 212, 25 (1990)

36. Instead of working in an Euler frame and applying an implicit time-integration scheme (1), we used a finitedifference solution of the LE in a Lagrangian frame in combination with an Euler time-integration scheme, resulting in a 1D MD description of the motion of jet slices along the propagation direction. The spatial resolution was made adaptable by splitting into two pieces the cylindrical Lagrange flow particles exceeding a certain length to radius ratio. The time step was adjusted according to the mass of the smallest flow particles, and two parts of the jet were disconnected whenever their connecting neck became $<2 \AA$. In the LE and SLE simulations, we used viscosity $\left(\eta=0.000011 \mathrm{Ns} / \mathrm{m}^{2}\right)$ and surface tension $(\gamma=0.009 \mathrm{~N} / \mathrm{m})$ values that were determined at a temperature of $210 \mathrm{~K}$ (i.e., the average molecular kinetic temperature in the jet obtained with the MD simulations) through separate MD simulations of liquid propane, and the velocity and velocity fluctuations obtained from the MD simulation of the jet [evaluated for the nonwetting nozzle case in a thin slice of the jet at $z=5 \mathrm{~nm}$, i.e., well beyond the velocity relaxation region (Fig. 2B)] were applied as in-flow initial conditions in these hydrodynamics simulations.

37. In (20) [see also (38)], it has been shown that the similarity solution (long threads) is unstable to finite amplitude perturbations, with the critical amplitude going to zero at the singularity, and it was concluded that thermal fluctuations in the liquid can trigger the instabilities. In the numerical simulations in (20) such fluctuations were introduced into the LE simulations in an ad hoc manner.

38. M. P. Brenner, X. D. Shi, S. R. Nagel, Phys. Rev. Lett. 73, 3391 (1994)

39. L. D. Landau and E. M. Lifshitz, Fluid Mechanics (Pergamon, Oxford, 1984)

40. R. F. Fox and G. Uhlenbeck, Phys. Fluids 13, 1893 (1970)

41. J. N. Israelachvili, Intermolecular and Surface Forces (Academic Press, London, 1991).

42. Supplemental material is available at Science Online at www.sciencemag.org/feature/data/1050049.shl.

43. J. B. Keller and M. J. Miksis, SIAM J. Appl. Math. 43 268 (1983)

44. SLE simulations where the inflow initial condition did not include fluctuations in the velocity yielded very similar results.

45. This research is supported by the U.S. Department of Energy, the Air Force Office of Scientific Research, and the Deutsche Forschungsgemeinschaft (to M.M.) We thank W. D. Luedtke and J. Gao for many useful conversations and R. F. Fox for bringing (40) to our attention. Simulations were performed on an IBM SP2 computer at the Georgia Tech Center for Computational Materials Science.

2 March 2000; accepted 6 June 2000 